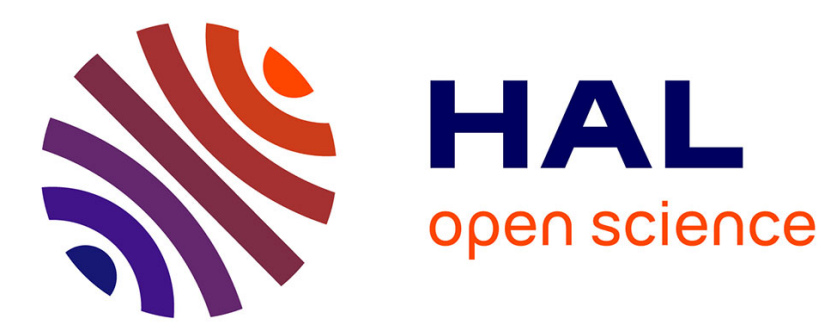

\title{
On the robustness analysis of triangular nonlinear systems: iISS and practical stability
}

\author{
Antoine Chaillet, Antonio Loria
}

\section{To cite this version:}

Antoine Chaillet, Antonio Loria. On the robustness analysis of triangular nonlinear systems: iISS and practical stability. IEEE International Multi-Conf. on Systems, Signals and Devices, Conf. on Systems, Analysis and Automatic Control, 2009, Djerba, Tunisia. hal-00441742

HAL Id: hal-00441742

https://hal-centralesupelec.archives-ouvertes.fr/hal-00441742

Submitted on 17 Dec 2009

HAL is a multi-disciplinary open access archive for the deposit and dissemination of scientific research documents, whether they are published or not. The documents may come from teaching and research institutions in France or abroad, or from public or private research centers.
L'archive ouverte pluridisciplinaire HAL, est destinée au dépôt et à la diffusion de documents scientifiques de niveau recherche, publiés ou non, émanant des établissements d'enseignement et de recherche français ou étrangers, des laboratoires publics ou privés. 


\title{
ON THE ROBUSTNESS ANALYSIS OF TRIANGULAR NONLINEAR SYSTEMS: IISS AND PRACTICAL STABILITY
}

\author{
Antoine Chaillet ${ }^{1}$ and Antonio Loría ${ }^{2}$ \\ ${ }^{1}$ EECI - L2S - Université Paris Sud - Supélec. \\ 3, rue Joliot-Curie. 91192 - Gif sur Yvette, France. chail let @ ieee. org \\ ${ }^{2}$ L2S - CNRS, same address. loriaelss. supelec.fr
}

\begin{abstract}
This note synthesizes recent results obtained by the authors on the stability and robustness analysis of cascaded systems. It focuses on two properties of interest when dealing with perturbed systems, namely integral input-tostate stability and practical stability. We present sufficient conditions for which each of these notions is preserved under cascade interconnection. The obtained conditions are of a structural nature, which makes their use particularly easy in practice.
\end{abstract}

Index Terms - Stability and robustness analysis, nonlinear systems, interconnected dynamical systems, cascades.

\section{INTRODUCTION}

The asymptotic stability analysis by Lyapunov's second method requires the construction of a strict Lyapunov function. This direct approach may be particularly hard for complex or large-scale nonlinear time-varying systems. A natural way of simplifying this problem consists in dividing the system into simpler interconnected subsystems, and to analyze each subsystem separately.

While a wide literature focuses on the feedback interconnection of dynamical subsystems, we concentrate here on recent results for the study of systems with a triangular or cascade form. Indeed, many applications can be represented as a unidirectional interconnection of dynamical subsystems.

Such a cascade structure often arises in the study of control systems. In particular, the so-called cascades-based design consists in designing a preliminary control law that makes the system have a cascade structure. This approach was followed in e.g. [6, 34] to control robot manipulators, where the mechanical (i.e. the robot arm) and the electrical (i.e. actuators) parts were addressed separately. See e.g. [33, 22, 25, 10, 18, 21, 44] for other examples of applications. Cascades stabilization was itself the subject of a great literature that we cannot cover here. We rather focus on the stability and robustness analysis of such systems. Indeed, in order to decompose a complex analysis

The iISS results presented here were established by the first author in collaboration with D. Angeli, Università di Firenze, Italy. into simpler problems using theorems for cascaded systems, it is crucial to know whether the stability properties of both subsystems taken separately remain valid for their cascade interconnection.

From a theoretical point of view, this problem is not trivial. It has attracted the interest of the control community since [27], where graph theory was used to ensure local and global stability properties of the cascade, based on the assumption that the interconnection terms are all "stability preserving mappings". In [50], converse Lyapunov results were used to show that uniform local asymptotic stability is naturally preserved by the cascade structure. Nevertheless, the global case presents harder difficulties. Intuitively, we could expect that, in order to preserve the global asymptotic stability of the cascade, it would suffice that the convergence rate of the driving subsystem be sufficiently high. This intuition is wrong in general, as proved in [45] through an elementary example involving a linear driving subsystem which yields a stronger peaking of the transients as the convergence is made faster. This transient peaking suffices to generate unbounded solutions. Similarly, as shown in [42, 47], neither integrability nor even exponential decay of the solutions of the driving subsystem is sufficient to preserve global asymptotic stability in general.

Beyond these obstacles, some sufficient conditions for the preservation of global asymptotic stability (GAS) under the cascade interconnection have been proposed in the literature. In general terms, a fundamental result for the analysis of global stability for nonlinear systems states that the cascade of GAS subsystems remains GAS if and only if its solutions are globally bounded. See [35, 38] for the proof of this statement in the case of autonomous systems and [32] for the case of time-varying systems.

Some work has then been done to advantageously replace the requirement of global boundedness of solutions by more structural conditions. In [35], these conditions take the form of a robustness Lyapunov condition on the driven subsystem that needs to hold for large values of the state. In [31, 26], uniform global boundedness of solutions is replaced by the requirements that the interconnection term be affine in the state of the driven subsystem, that the solutions of the driving subsystem be exponentially converging (or at least integrable) and that a Lyapunov func- 
tion, with a convenient bound on its gradient be known for the driven subsystem. In [32], further sufficient conditions are provided, expressed as dominance relationships linking the interconnection and drift terms. In [4], an elegant reformulation of the integrability condition posed in $[31,32]$ is established in terms of integral input-to-state stability (iISS ${ }^{1}$ ). More precisely, that reference proposes a condition linking the dissipation rate of the driving subsystem to the iISS gain of the driven under which the cascade interconnection of an iISS system driven by a GAS one is itself GAS. As iISS is a much weaker property than inputto-state stability (ISS) [39, 40], that result thus strongly relaxes the well known fact that a cascade of GAS subsystems is GAS if the driven subsystem is ISS, this fact being itself a direct consequence of the natural preservation of ISS under cascade interconnection [43]. The same results are highlighted from a different perspective in [31, 32].

Although very rich, most of this literature is concerned with the obtention of asymptotic stability properties for the overall cascade. However, when disturbances are considered (e.g. non-vanishing perturbations, model uncertainty or measurement imprecision), convergence to the origin may be impeded (steady-state error). This property is referred to as ultimate boundedness, cf. e.g. [14, 51]. In some practical circumstances, the operating point of a given system may be mathematically unstable, thus generating small oscillations around it, but still guarantee a sufficient precision for an acceptable behavior. This is especially true when feedback gains, or other free design parameters, may be tuned to arbitrarily reduce the amplitude of the steady-state error. This stronger property is referred to as practical stability. It will be the main subject of Section 3, in which we will recall the notion of global practical stability for nonlinear time-varying systems and present a growth rate condition, originally derived in [6], under which it is preserved by cascade interconnection.

The other series of results we recall in this note addresses a similar question for the iISS property. It is well known [4] that, contrarily to ISS, iISS is not preserved by cascade interconnection. We therefore provide conditions under which this preservation holds. These conditions are first given in the case when an explicit iISS Lyapunovlike function is known for each of the two subsystems. Roughly, it suffices that the dissipation term of the driving subsystem dominates the supply function of the driven one in a neighborhood of the origin. The second step consists in stating this condition in terms of the estimates of the trajectories of the two subsystems when disconnected. More precisely, in the case of a continuously differentiable zero-input driving subsystem, we impose, similarly to [4], that the driven subsystem presents a locally Lipschitz iISS gain, and the driving one be locally exponentially stable when no disturbance applies.

In addition, we complete the main result in [4] by giving a sufficient condition for the cascade composed of an iISS driven by a GAS one to remain GAS in the case when explicit Lyapunov functions are known. Roughly,

${ }^{1}$ The definition of this property is recalled in the sequel. it is again required that the dissipation term of the GAS subsystem dominates the supply function of the iISS one around zero. This result may be useful in practice since the iISS and GAS properties are commonly established through Lyapunov arguments. Furthermore, this result naturally extends to multiple cascaded systems, i.e. series of cascaded iISS systems driven by a GAS one. These results, originally presented in [5], are all presented in Section 2 .

Notation: Before continuing, we introduce the notation we will make use of along these lines. $\mathcal{P} \mathcal{D}$ denotes the class of all continuous positive definite functions $\mathbb{R}_{\geq 0} \rightarrow$ $\mathbb{R}_{>0}$. $\mathcal{K}$ designates the set of all continuous increasing functions $\mathbb{R}_{\geq 0} \rightarrow \mathbb{R}_{\geq 0}$ that vanish at 0 . A function is said to belong to class $\mathcal{K}_{\infty}$ if is of class $\mathcal{K}$ and tends to infinity with its argument. $\mathcal{L}$ is the class of all continuous decreasing functions $\mathbb{R}_{\geq 0} \rightarrow \mathbb{R}_{\geq 0}$ that tend to zero when their argument tends to infinity. A function is said to be in class $\mathcal{K} \mathcal{L}$ if it is of class $\mathcal{K}$ in their first argument and of class $\mathcal{L}$ in the second argument. Given any positive $\varepsilon$, the set of all functions $\alpha$ satisfying $\lambda \alpha(a+b) \leq \alpha(a)+\alpha(b)$ for all $a, b \in(0, \varepsilon)$ and for some constant $\lambda>0$ is referred to as $\mathcal{I}_{\varepsilon}$. Given a positive $\delta, \mathcal{B}_{\delta}$ denotes the open ball of radius $\delta$, centered at the origin, in the Euclidean space of appropriate dimension. Let $a \in\{0,+\infty\}$ and $q_{1}$ and $q_{2}$ be class $\mathcal{K}$ functions. We say that $q_{1}$ dominates $q_{2}$ in a neighborhood of $a$ (and we write $q_{2}(s)=$ $\mathcal{O}\left(q_{1}(s)\right)$ if there exists a nonnegative constant $k$ such that $\limsup _{s \rightarrow a} q_{2}(s) / q_{1}(s) \leq k$. We say that $q_{1}$ strictly dominates $q_{2}$ (notation: $\left.q_{2}(s)=o\left(q_{1}(s)\right)\right)$ if $k$ can be taken as 0 , and that $q_{1}$ is equivalent to $q_{2}$ (i.e., $q_{1}(s) \sim q_{2}(s)$ ) if $\lim _{s \rightarrow a} q_{2}(s) / q_{1}(s)=1$. $\llbracket a, b \rrbracket$ denotes the set of all integers in $[a, b]$. A dynamical system is said to be GAS if its origin is globally asymptotically stable. It is said to be LES if its origin is locally exponentially stable.

\section{CASCADES INVOLVING IISS SYSTEMS}

The results of this section, originally presented in [5], deal with nonlinear systems on which an input $u$ is applied. We assume for simplicity that the only possible time-dependency occurs through the applied input. In other words, the class studied in this section encompasses all systems of the form

$$
\dot{x}=f(x, u)
$$

where $x \in \mathbb{R}^{n}$ is the state and $f: \mathbb{R}^{n} \times \mathbb{R}^{m} \rightarrow \mathbb{R}^{n}$ is a locally Lipschitz function. Input signals $u$ are assumed to be in the class $\mathcal{U}$, made of all measurable locally essentially bounded functions $u: \mathbb{R}_{\geq 0} \rightarrow \mathbb{R}^{m}$.

The study of the impact of the input $u$ on the overall behavior of the plant has been the subject of a rich literature. A particularly powerful framework to this respect is that of input-to-state stability (ISS) and its declinations; see [41] for a survey on this notion. The ISS property imposes that solutions be bounded by a fading function of the initial state plus a term that is somewhat "proportional" to the amplitude of the applied input. A generalization of this 
property, involving a measure of the energy that the input feeds to the system (rather than its amplitude) is known as integral input-to-state stability (iISS). This notion happens to be a much wider concept than ISS and will be the key tool for the cascade analysis conducted here.

\subsection{Related definitions}

We start by recalling the definition of iISS itself and some properties that this concept naturally induces, as well as related notions.

Definition 1 (iISS, [40]) We say that (1) is integral inputto-state stable with respect to $u$ if there exist functions $\beta \in$ $\mathcal{K} \mathcal{L}$ and $\gamma, \mu \in \mathcal{K}$ such that, for all $x_{0} \in \mathbb{R}^{n}$ and all $u \in \mathcal{U}$, its solution $x\left(\cdot ; x_{0}, u\right)$ satisfies, for all $t \in \mathbb{R}_{\geq 0}$,

$$
\left|x\left(t ; x_{0}, u\right)\right| \leq \beta\left(\left|x_{0}\right|, t\right)+\gamma\left(\int_{0}^{t} \mu(|u(\tau)|) d \tau\right) .
$$

The function $\mu$ is then referred to as an iISS gain for (1).

As established in [3], iISS is more conservative than requiring that the zero-input $\dot{x}=f(x, 0)$ be GAS and that $\dot{x}=f(x, u)$ be forward complete. Yet, it holds very often in specific applications involving GAS systems disturbed by external signals. Specific control design methods, such as [24], may also induce this property by state feedback.

While very general, this property induces interesting robustness properties on the perturbed system (1). For instance, it is shown in [40, Proposition 6] that, for any input $u$ such that $\int_{0}^{\infty} \mu(|u(\tau)|) d \tau$ is finite, the solution of an iISS system with iISS gain $\mu$ eventually converges to zero.

It was established in [3] that a necessary and sufficient condition for a system like (1) to be iISS is that there exist a positive definite radially unbounded continuously differentiable function $V: \mathbb{R}^{n} \rightarrow \mathbb{R}_{\geq 0}$, a $\mathcal{K}$ function $\gamma$ and a $\mathcal{P D}$ function $\alpha$ satisfying, for all $x \in \mathbb{R}^{n}$ and all $u \in \mathbb{R}^{m}$,

$$
\frac{\partial V}{\partial x}(x) f(x, u) \leq-\alpha(|x|)+\gamma(|u|)
$$

This Lyapunov characterization is very similar to its ISS analogous, at the exception that $\alpha$ is required to be a $\mathcal{K}_{\infty}$ function for the latter.

We also recall that the system (1) is said to be 0-GAS (resp. 0-LES) if the origin of $\dot{x}=f(x, 0)$ is GAS (resp. LES). It is said to satisfy the bounded energy - frequently bounded state (BEFBS) property if there exists $\sigma \in \mathcal{K}_{\infty}$ such that, for all $x_{0} \in \mathbb{R}^{n}$,

$\int_{0}^{\infty} \sigma(|u(\tau)|) d \tau<\infty \Rightarrow \liminf _{t \rightarrow+\infty}\left|x\left(t ; x_{0}, u\right)\right|<\infty$

It was shown in [2] that the combination of 0-GAS and BEFBS is equivalent to iISS. This observation is at the basis of the proof of most results presented in this section.

\subsection{Lyapunov-based condition for cascades composed of an iISS system driven by a GAS one}

The first result we recall here is concerned with cascades composed of an iISS subsystem driven by a GAS one. It therefore focuses on systems of the form

$$
\begin{aligned}
& \dot{x}_{1}=f_{1}\left(x_{1}, x_{2}\right) \\
& \dot{x}_{2}=f_{2}\left(x_{2}\right),
\end{aligned}
$$

where $x_{1} \in \mathbb{R}^{n_{1}}, x_{2} \in \mathbb{R}^{n_{2}}$ and the functions $f_{1}$ and $f_{2}$ are both assumed to be locally Lipschitz. We also assume that $f_{1}(0,0)=0$ and $f_{2}(0)=0$.

The first result we present here establishes a sufficient condition for a cascade of an iISS subsystem driven by a GAS one to be GAS: this conditions takes the form of an order comparison between the dissipation rate $\alpha_{2}$ of the driving subsystem and the iISS gain $\gamma_{1}$ of the driven one.

Theorem 1 (GAS + iISS) Assume that the origin of (2b) is globally attractive ${ }^{2}$ and that there exist a constant $\varepsilon>0$ and two continuous positive definite radially unbounded functions $V_{1}$ and $V_{2}$, differentiable on $\mathbb{R}^{n_{1}}$ and $\mathcal{B}_{\varepsilon} \backslash\{0\}$ respectively, and satisfying

$$
\begin{gathered}
\frac{\partial V_{1}}{\partial x_{1}} f_{1}\left(x_{1}, x_{2}\right) \leq-\alpha_{1}\left(\left|x_{1}\right|\right)+\gamma_{1}\left(\left|x_{2}\right|\right), \forall\left(x_{1}, x_{2}\right) \in \mathbb{R}^{n_{1}+n_{2}} \\
\frac{\partial V_{2}}{\partial x_{2}} g\left(x_{2}\right) \leq-\alpha_{2}\left(\left|x_{2}\right|\right), \quad \forall x_{2} \in \mathcal{B}_{\varepsilon} \backslash\{0\}
\end{gathered}
$$

where $\alpha_{1}, \alpha_{2} \in \mathcal{P D}$ and $\gamma_{1} \in \mathcal{K}$. Assume further that

$$
\gamma_{1}(s)=\mathcal{O}\left(\alpha_{2}(s)\right), \quad \text { as } \quad s \rightarrow+\infty .
$$

Then the cascade (2) is GAS.

Note that such a result, i.e. studying the cascade interconnection of an iISS subsystem driven by a GAS one, was also the purpose of [4]. The main difference here is that the GAS of the driving subsystem and the iISS of the driven one are not established through an explicit estimate of their solutions, but rather in terms of Lyapunov functions which constitute a natural tool for establishing these properties.

To this respect, note that the combination of global attractivity and (4) is actually equivalent to GAS of (2b). The above statement is motivated by an easier applicability in practice and the possibility it offers for generalizing to multiple cascades, as we will see later on in Theorem 2.

While authorizing a slight additional flexibility, as shown in the sequel, the fact of not requiring the differentiability of $V_{2}$ at zero is motivated by homogeneity concerns with Theorem 4. The Lyapunov-like function constructed for the proof of the latter is indeed possibly non-differentiable at the origin.

A similar result can be derived from the recent contributions of H. Ito: $[11,12,13]$. In those references, a small gain condition for iISS systems is provided and, as a particular case, cascades composed of an iISS system

\footnotetext{
${ }^{2}$ i.e., the solution of $(2 \mathrm{~b})$ tends to the origin from any initial state.
} 
driven by a GAS one are considered. The condition imposed there, however, involves the $\mathcal{K}_{\infty}$ bounds on $V_{1}$ and is expressed as a dominance condition on the whole state space, whereas the one presented here (5) needs to hold only in a neighborhood of the origin zero. Also, the conditions in those references implicitly require that the dissipation rate $\alpha_{2}$ of the driving subsystem be of class $\mathcal{K}$, as this has been shown to be necessary for a general small gain theorem of not-necessarily ISS systems [1]. While those results apply to a more general context (feedback interconnection), these two features make the dominance condition (5) less conservative and easier to apply as far as cascades are concerned.

Remark 1 (Relaxation of (5)) It is worth mentioning that, if an upper bound on $V_{2}$ of the form $V_{2}\left(x_{2}\right) \leq \bar{\alpha}_{2}\left(\left|x_{2}\right|\right)$ is explicitly known, where $\bar{\alpha}_{2}$ designates a $\mathcal{K}_{\infty}$ function, condition (5) can be relaxed to the existence of a constant $p \in[0,1)$ such that

$$
\gamma_{2}(s)=\mathcal{O}\left(\frac{\alpha_{2}(s)}{\bar{\alpha}_{2}(s)^{p}}\right), \alpha_{2}(s)=o\left(\bar{\alpha}_{2}(s)^{p}\right) \text {, as } s \rightarrow 0 .
$$

Indeed, consider the function $\mathcal{V}_{2}(\cdot):=V_{2}(\cdot)^{1-p}$. Then $\mathcal{V}_{2}$ is a positive definite radially unbounded function, differentiable on $\mathbb{R}^{n_{2}} \backslash\{0\}$, and we get from (4) that

$$
\begin{aligned}
\frac{\partial \mathcal{V}_{2}}{\partial x_{2}}\left(x_{2}\right) f_{2}\left(x_{2}\right) & \leq-(1-p) \alpha_{2}\left(\left|x_{2}\right|\right) V_{2}^{-p}\left(x_{2}\right) \\
& \leq-(1-p) \frac{\alpha_{2}\left(\left|x_{2}\right|\right)}{\bar{\alpha}_{2}\left(\left|x_{2}\right|\right)^{p}}=:-\tilde{\alpha}_{2}\left(\left|x_{2}\right|\right) .
\end{aligned}
$$

In view of (6), $\tilde{\alpha}_{2} \in \mathcal{P} \mathcal{D}$. Hence Theorem 1 applies with $\mathcal{V}_{2}$, and establishes that (2) is GAS. In this respect, notice that allowing $V_{2}$ to be non-differentiable at the origin is useful, as further illustrated by the following example.

Example 1 Consider the following two-dimensional system, consisting in a particular case of [4, Example 4$]^{3}$ :

$$
\begin{aligned}
& \dot{x}_{1}=-\operatorname{sat}\left(x_{1}\right)+x_{1} x_{2} \\
& \dot{x}_{2}=-\frac{x_{2}}{1+x_{2}^{2}},
\end{aligned}
$$

where sat $(r):=\operatorname{sign}(r) \min \{1,|r|\}$ for all $r \in \mathbb{R}$. Direct computations show that the functions $V\left(x_{1}\right)=\frac{1}{2} \ln (1+$ $\left.x_{1}^{2}\right)$ and $V_{2}\left(x_{2}\right)=\frac{1}{2} x_{2}^{2}$ satisfy (3) and (4) with $\alpha_{1}(s)=$ $($ s sats $) /\left(1+s^{2}\right), \gamma_{2}(s)=s$ and $\alpha_{2}(s)=s^{2} /\left(1+s^{2}\right)$. Since the requirement $\gamma_{2}(s)=\mathcal{O}\left(\alpha_{2}(s)\right)$ as $s$ tends to zero does not hold, it is not possible to apply Theorem 1 directly. Nevertheless, it is possible to conclude GAS using the previous remark with $p=1 / 2$. Indeed, take $\bar{\alpha}\left(\left|x_{2}\right|\right)=$ $\frac{1}{2}\left|x_{2}\right|^{2} \geq V_{2}\left(x_{2}\right)$, then $\bar{\alpha}_{2}(s)^{p}=s / \sqrt{2}$ strictly dominates $\alpha_{2}(s)$ around zero, and $\alpha_{2}(s) / \bar{\alpha}_{2}(s)^{p}=s \sqrt{2} /\left(1+s^{2}\right)$ dominates $\gamma_{2}(s)$ for small $s$.

We stress that, although the present use of Lyapunovbased conditions simplifies the argument, the above example can also be addressed by other methods such as $[4,32]$.

\footnotetext{
${ }^{3}$ It also appears in [32] and was originally suggested by Laurent Praly.
}

\subsection{Lyapunov-based conditions for GAS of multiple systems in cascades}

While Theorem 1 is stated for the cascade interconnection of two nonlinear subsystems, it easily extends to multiple cascades, i.e.

$$
\begin{aligned}
\dot{x}_{1} & =f_{1}\left(x_{1}, x_{2 \rightarrow N}\right) \\
\dot{x}_{2} & =f_{2}\left(x_{2}, x_{3 \rightarrow N}\right) \\
& \vdots \\
\dot{x}_{N-1} & =f_{N-1}\left(x_{N-1}, x_{N}\right) \\
\dot{x}_{N} & =f_{N}\left(x_{N}\right),
\end{aligned}
$$

where we used the notation $x_{i \rightarrow j}$ to denote $\left(x_{i}^{T}, \ldots, x_{j}^{T}\right)^{T} \in$ $\mathbb{R}^{n_{i}+\ldots+n_{j}}$ for all integers $0 \leq i \leq j$. The functions $f_{i}: \mathbb{R}^{n_{i}} \times \mathbb{R}^{n_{i}+\ldots N} \rightarrow \mathbb{R}^{n_{i}}$ are all assumed to be locally Lipschitz and to vanish at the origin.

The following result establishes GAS of (7) by assuming that ${ }^{4}(7 . N)$ is itself GAS and that all subsystems (7.i), $i \in \llbracket 1, N-1 \rrbracket$, are iISS. It relies on growth conditions (8) on the supply functions involved in the Lyapunov characterization of each subsystem involved. The proof requires a particular behavior of the involved supply functions in a neighborhood of zero. Namely, it imposes that they all belong to the class $\mathcal{I}_{\varepsilon}$, for some $\varepsilon>0$, as defined in the notation section.

Theorem 2 (GAS+iISS+iISS+. . .) Assume that the origin of (7.N) is globally attractive and that there exist a constant $\varepsilon>0$ and, for each $i \in \llbracket 1, N \rrbracket$, a continuous positive definite radially unbounded function $V_{i}$, differentiable on $\mathbb{R}^{n_{i}}$, and satisfying, for all $i \in \llbracket 1, N-1 \rrbracket$,

$$
\begin{gathered}
\frac{\partial V_{i}}{\partial x_{i}} f_{i}\left(x_{i}, x_{i+1 \rightarrow N}\right) \leq-\alpha_{i}\left(\left|x_{i}\right|\right)+\gamma_{i}\left(\left|x_{i+1 \rightarrow N}\right|\right), \forall x_{i} \in \mathbb{R}^{n_{i}} \\
\frac{\partial V_{N}}{\partial x_{N}} f_{N}\left(x_{N}\right) \leq-\alpha_{N}\left(\left|x_{N}\right|\right), \quad \forall x_{N} \in \mathcal{B}_{\varepsilon} \backslash\{0\},
\end{gathered}
$$

where, for each $i \in \llbracket 1, N \rrbracket, \alpha_{i} \in \mathcal{P} \mathcal{D} \cap \mathcal{I}_{\varepsilon}$ and, for each $i \in \llbracket 1, N-1 \rrbracket, \gamma_{i} \in \mathcal{K} \cap \mathcal{I}_{\varepsilon}$. Then, under the condition that

$$
\begin{array}{rlrl}
\gamma_{i}(s) & =\mathcal{O}\left(\alpha_{i+1}(s)\right), & & \forall i \in \llbracket 1, N-1 \rrbracket \\
\gamma_{i}(s)=\mathcal{O}\left(\gamma_{i+1}(s)\right), & & \forall i \in \llbracket 1, N-2 \rrbracket,
\end{array}
$$

as $s$ tends to zero, the cascade (7) is GAS.

Remark 2 (Differentiability of $V_{N}$ ) Although not stated explicitly for the sake of compactness, $V_{N}$ is only required to be differentiable over $\mathcal{B}_{\varepsilon} \backslash\{0\}$.

The proof of the above result is detailed in [5]. Note that the requirement that all supply functions be in the class $\mathcal{I}_{\varepsilon}$ is little conservative in practice as it needs to hold only locally. For instance, it is satisfied by any function with polynomial behavior around zero.

\footnotetext{
${ }^{4} \mathrm{We}$ use the notation (7.i) to denote the system $\dot{x}_{i}=$ $f_{i}\left(x_{i}, x_{i+1 \rightarrow N}\right)$.
} 


\subsection{Lyapunov-based condition for cascades composed of two iISS systems}

The next result studies the cascade connection of two iISS systems, in the case when an iISS-Lyapunov function is explicitly known for each of them. For the sake of generality, it is allowed that the driven subsystem depends also on the external input. We therefore deal with systems of the form:

$$
\begin{aligned}
& \dot{x}_{1}=f_{1}\left(x_{1}, x_{2}, u\right) \\
& \dot{x}_{2}=f_{2}\left(x_{2}, u\right)
\end{aligned}
$$

where $x_{1} \in \mathbb{R}^{n_{1}}, x_{2} \in \mathbb{R}^{n_{2}}, u: \mathbb{R}_{\geq 0} \rightarrow \mathbb{R}^{m}$ is measurable locally essentially bounded, $f_{1}$ and $f_{2}$ are locally Lipschitz and satisfy $f_{1}(0,0,0)=0$ and $f_{2}(0,0)=0$.

Theorem 3 (iISS+iISS) Let $V_{1}$ and $V_{2}$ be continuous positive definite radially unbounded functions, differentiable on $\mathbb{R}^{n_{1}}$ and $\mathbb{R}^{n_{2}}$ respectively. Suppose that there exist $\nu_{1} \in \mathcal{K}$ and, for all $i \in\{1,2\}, \alpha_{i} \in \mathcal{P} \mathcal{D}$ and $\gamma_{i} \in \mathcal{K}$ such that, for all $\left(x_{1}, x_{2}\right) \in \mathbb{R}^{n_{1}} \times \mathbb{R}^{n_{2}}$ and all $u \in \mathbb{R}^{m}$,

$$
\begin{aligned}
& \frac{\partial V_{1}}{\partial x_{1}} f_{1}\left(x_{1}, x_{2}, u\right) \leq-\alpha_{1}\left(\left|x_{1}\right|\right)+\gamma_{1}\left(\left|x_{2}\right|\right)+\nu_{1}(|u|) \\
& x_{2} \neq 0 \Rightarrow \frac{\partial V_{2}}{\partial x_{2}} f_{2}\left(x_{2}, u\right) \leq-\alpha_{2}\left(\left|x_{2}\right|\right)+\gamma_{2}(|u|) .
\end{aligned}
$$

Then the cascade (9) is iISS provided that

$$
\gamma_{1}(s)=\mathcal{O}\left(\alpha_{2}(s)\right), \quad \text { as } \quad s \rightarrow 0 \text {. }
$$

See [5] for the proof of this result. We stress that, unlike Theorem 1 , the above result is not easily extendable to cascades involving more than two subsystems as condition (11) is required to hold on the whole $\mathbb{R}^{n_{2}} \backslash\{0\}$ and not only locally as (4), which makes the dissipation rate resulting from the application of Theorem 3 difficult to estimate.

A direct consequence of Theorem 3, which is of notable interest in practice, concerns the case when the driven subsystem does not depend on the input $u$. The system then takes the more classical cascade form

$$
\begin{aligned}
& \dot{x}_{1}=f_{1}\left(x_{1}, x_{2}\right) \\
& \dot{x}_{2}=f_{2}\left(x_{2}, u\right) .
\end{aligned}
$$

Corollary 1 Let $V_{1}$ and $V_{2}$ be as in Theorem 3 and suppose that, for all $i \in\{1,2\}$, there exist $\alpha_{i} \in \mathcal{P} \mathcal{D}$ and $\gamma_{i} \in \mathcal{K}$ such that, for all $\left(x_{1}, x_{2}\right) \in \mathbb{R}^{n_{1}} \times \mathbb{R}^{n_{2}}$, (11) holds and

$$
\frac{\partial V_{1}}{\partial x_{1}}\left(x_{1}\right) f_{1}\left(x_{1}, x_{2}, u\right) \leq-\alpha_{1}\left(\left|x_{1}\right|\right)+\gamma_{1}\left(\left|x_{2}\right|\right) .
$$

Then the cascade (13) is iISS provided that (12) holds.

Intuitively, one could expect that the cascade inherits the iISS gain of its driving subsystem. Example 8 in [5] show that this intuition is not true in general.

\subsection{Trajectory-based condition for cascades of iISS sys-} tems

The last two results of this section propose a sufficient condition for a cascade of iISS systems to remain iISS, without requiring the knowledge of any Lyapunov function. Instead, greater stability properties are required for the subsystems involved. It is indeed imposed that the origin of the driving subsystem be locally exponentially stable when no input is applied, and that the iISS gain of the driven subsystem be locally Lispchitz. See [5, Section 4.1] for the proof.

Theorem 4 (iISS + iISS, trajectory-based) Assume that $f_{2}(\cdot, 0)$ is continuously differentiable. Assume that the system $(9 a)$ is iISS with respect to $\left(x_{2}^{T}, u^{T}\right)^{T}$ with an locally Lispchitz iISS gain, and that the system (9b) is iISS and O-LES. Then, the cascade (9) is iISS.

It is worth noting that the condition we recover here is similar to the one derived from [4] for cascades constituted of an iISS subsystem driven by a GAS one. Condition (10) in [4] is indeed naturally fulfilled when the iISS gain is locally Lipschitz and the origin of the driving subsystem is locally exponentially stable.

Also, similarly to Theorem 3, note that this result applies to cascaded systems like (13), i.e. when the driven subsystem does not depend on $u$.

Under additional regularity conditions on the dynamics of the driven subsystems, the above result may extend to multiple cascaded systems, i.e.

$$
\begin{aligned}
\dot{x}_{1} & =f_{1}\left(x_{1}, \zeta_{2 \rightarrow N}\right) \\
\dot{x}_{2} & =f_{2}\left(x_{2}, \zeta_{3 \rightarrow N}\right) \\
& \vdots \\
\dot{x}_{N-1} & =f_{N-1}\left(x_{N-1}, \zeta_{N \rightarrow N}\right) \\
\dot{x}_{N} & =f_{N}\left(x_{N}, u\right),
\end{aligned}
$$

where $\zeta_{i \rightarrow N}:=\left(x_{i}^{T}, \ldots, x_{N}^{T}, u^{T}\right)^{T} \in \mathbb{R}^{n_{i}+\ldots+n_{N}+m}$, for all $i \in \llbracket 2, N \rrbracket$. By convention, $\zeta_{N+1 \rightarrow N}:=u$. The following result was proved in [5, Section 4.5].

\section{Theorem 5 (Cascade of multiple iISS systems)}

Assume that, for each $i \in \llbracket 1, N \rrbracket$ the subsystem (14.i) is 0 -LES and iISS with respect to $\zeta_{i+1 \rightarrow N}$. Assume also that, for each $i \in \llbracket 1, N-1 \rrbracket$, the function $f_{i}(\cdot, 0)$ is continuously differentiable, $\partial f_{i}(\cdot, 0) / \partial x_{i}$ is bounded in a neighborhood of the origin and the iISS gain of (14.i) is locally Lipschitz. Then the cascade (14) is iISS.

\section{GLOBAL PRACTICAL ASYMPTOTIC STABILITY}

\subsection{Definition and Lyapunov characterization}

The second part of this paper synthesizes recent results for the robustness analysis of cascades composed of parameterized nonlinear time-varying subsystems of the form

$$
\dot{x}=f(t, x, \theta) \text {, }
$$


where $x \in \mathbb{R}^{n}$ is the state, $t \in \mathbb{R}_{\geq 0}$ is the time, $\theta \in \mathbb{R}^{m}$ is a constant free parameter and $f: \mathbb{R}_{\geq 0} \times \mathbb{R}^{n} \times \mathbb{R}^{m} \rightarrow \mathbb{R}^{n}$ is locally Lipschitz in $x$ and satisfies Carathéodory conditions for all $\theta$ under consideration.

When (15) is a plant in closed-loop with a state feedback control law, the parameter $\theta$ typically contains control gains ( $c f$. e.g. $[30,7])$. But $\theta$ may also represent other design parameters cf. e.g. [49, 46, 48, 23, 20].

Contrarily to the previous section, we here consider the possible explicit time-dependency of the plant dynamics. While this time-dependency was implicitly contained in the input $u$ applied to the systems studied in Section 2, the present section does not explicitly consider these inputs. The time-dependency of (15) may arise from the application of such an exogenous signal, but it may result from other causes such as trajectory tracking, control of plants that are not stabilizable by continuous time-invariant feedback $^{5}$ or systems with time-varying parameters. While the tools we present in the sequel allow consequently for a time-dependency of the Lyapunov functions involved, the properties we derive are all uniform in the initial time, meaning that the overall behavior of the solutions of (15) is independent of the initial time.

Definition 2 (UGPAS) Let $\Theta \subset \mathbb{R}^{m}$ be a set of parameters. The system (15) is said to be uniformly globally practically asymptotically stable on $\Theta$ if, given any positive $\delta$, there exists $\theta^{\star}(\delta) \in \Theta$ and $\beta_{\delta} \in \mathcal{K} \mathcal{L}$ such that, for any $x_{0} \in \mathbb{R}^{n}$ and any $t_{0} \in \mathbb{R}_{\geq 0}$, the solution of $\dot{x}=f\left(t, x, \theta^{\star}\right)$ satisfies

$$
\left|x\left(t ; t_{0}, x_{0}, \theta^{\star}\right)\right| \leq \delta+\beta_{\delta}\left(\left|x_{0}\right|, t-t_{0}\right), \quad \forall t \geq t_{0} .
$$

Hence, roughly speaking, we say that (15) is UGPAS if the size of the ball to which solutions converge can be arbitrarily diminished by a convenient choice of $\theta$. In other words, although asymptotic convergence to zero may be impeded $^{6}$, the precision reached by the plant can be made arbitrarily tight by the proper use of control parameters. Such a situation is fairly common in control practice, notably in the case of UGAS controlled systems perturbed by bounded external disturbances.

The above notion of practical stability should not be confused with existing definitions in the literature that correspond more to the concept of ultimate boundedness (see e.g. [14]), in the sense that they require that solutions eventually enter a ball without leaving it anymore but do not impose that this ball be reducible at will by tuning a parameter.

Other definitions are more conservative than Definition 2 , as they require that the $\mathcal{K} \mathcal{L}$ estimate, or at least its dependency on the initial state, be uniform in the parameters $\theta \in \Theta$ (not allowing $\beta$ to depend on the precision $\delta$ one wants to reach). While the latter property is satisfied in many contexts (see e.g. [49, 28, 46]), it may fail when

\footnotetext{
${ }^{5}$ It was shown in [9] that any completely controllable smooth system without drift (including nonholonomic mechanical systems) can be stabilized by means of a smooth periodic time-varying state feedback.

${ }^{6}$ The origin may not even be an equilibrium.
}

dealing with perturbed systems: the transients overshoot being often linked to the control gains (see [7] for an example in robot control). It should therefore be clear that, in Definition 2, "uniform" refers only to the initial conditions, and not to the tuning parameter.

Finally, we stress that other definitions, such as in [49, 29], require that the tuning parameter be a positive scalar that needs to be diminished in order to get a better precision, in which case any smaller parameter is guaranteed to induce at least the same precision. No such tuning procedure is imposed by Definition 2 as the parameter $\theta$ does not need to be scalar, making possible to take into account multiple gains tuning (see e.g. $[8,19,16]$ for applications involving mechanical systems).

The following result, proved in [6, Section IV-A], gives a sufficient condition, in terms of a Lyapunov function defined out of a neighborhood of the origin, for the dynamical system (15) to be uniformly globally practically asymptotically stable on a given set of parameters.

\section{Proposition 1 (Lyapunov condition for UGPAS)}

Suppose that, given any $\delta>0$, there exist a parameter $\theta(\delta) \in \Theta$, a continuously differentiable Lyapunov function $V_{\delta}: \mathbb{R}_{\geq 0} \times \mathbb{R}^{n} \rightarrow \mathbb{R}_{\geq 0}$ and class $\mathcal{K}_{\infty}$ functions $\underline{\alpha}_{\delta}, \bar{\alpha}_{\delta}$, $\alpha_{\delta}$ such that, for all $x \in \mathbb{R}^{n} \backslash \mathcal{B}_{\delta}$ and all $t \in \mathbb{R}_{\geq 0}$,

$$
\begin{gathered}
\underline{\alpha}_{\delta}(|x|) \leq V_{\delta}(t, x) \leq \bar{\alpha}_{\delta}(|x|) \\
\frac{\partial V_{\delta}}{\partial t}(t, x)+\frac{\partial V_{\delta}}{\partial x}(t, x) f(t, x, \theta) \leq-\alpha_{\delta}(|x|) .
\end{gathered}
$$

Then the system (15) is UGPAS on the parameter set $\Theta$ provided that the following relation holds:

$$
\lim _{\delta \rightarrow 0} \underline{\alpha}_{\delta}^{-1} \circ \bar{\alpha}_{\delta}(\delta)=0 .
$$

Compared to classical results for Lyapunov stability, conditions (16) and (17) are natural: they are similar to the Lyapunov sufficient condition for global ultimate boundedness ( $c f$. e.g. [14]). Intuitively, one may expect that these two requirements, when valid for any arbitrarily small tolerance $\delta$, suffice to conclude UGPAS. However, an additional assumption (18) is required that links the bounds on the Lyapunov function. Indeed, as opposed to previously cited definitions of practical stability, the Lyapunov function may depend on the tuning parameter $\theta$, and consequently on the desired precision $\delta$. As clearly shown in $[36,15]$, this parametrization of the Lyapunov function may induce unexpected behaviors of solutions if (18) is violated.

We finally stress that, although not included in the present paper, similar results as those presented here were derived for semiglobal stability. This situation refers to the case when the domain of attraction is not the whole state space but rather a compact neighborhood of the origin whose size can be enlarged at will by conveniently tuning the control parameters. We refer the reader to [7] for details on these aspects.

In many applications, the requirement (18) that links the lower and upper $\mathcal{K}_{\infty}$ bounds on the Lyapunov function 
follows from the combination of three properties: these bounds are affine in the tuning parameters $\theta$, they are polynomial functions of the same degree, and the parameters are affine in the inverse of the radius $\delta$ of the attractive ball. We therefore recall the following result that especially fits this situation. Although less general, it is more easily applicable. See $[16,19]$ for applications of this corollary in control of a spacecraft formation and for the automatic positioning of ships for underway replenishment.

\section{Corollary 2 (Simplified condition for UGPAS)}

Let $\Theta$ be a subset of $\mathbb{R}^{n}$. Assume that there exist a positive constant $p$, real constants $\underline{a}_{i}, \bar{a}_{i}, \underline{b}_{i}, \bar{b}_{i}, i \in \llbracket 1, n \rrbracket$ and, for any $\theta \in \Theta$, a continuously differentiable Lyapunov function $V_{\theta}$ satisfying, for all $x \in \mathbb{R}^{n}$ and all $t \in \mathbb{R}_{\geq 0}$,

$$
\sum_{i=1}^{n}\left(\underline{a}_{i}+\underline{b}_{i} \theta_{i}\right)\left|x_{i}\right|^{p} \leq V_{\theta}(t, x) \leq \sum_{i=1}^{n}\left(\bar{a}_{i}+\bar{b}_{i} \theta_{i}\right)\left|x_{i}\right|^{p}
$$

where, for all $i \in \llbracket 1, n \rrbracket$ and all $\theta \in \Theta, \underline{a}_{i}+\underline{b}_{i} \theta_{i}>0$ and $\bar{a}_{i}+\bar{b}_{i} \theta_{i}>0$. Suppose further that, given any $\delta>0$, there exist a parameter $\theta^{\star}(\delta) \in \Theta$ and a class $\mathcal{K}_{\infty}$ function $\alpha_{\delta}$ such that, for all $x$ such that $|x| \geq \delta$ and all $t \in \mathbb{R}_{\geq 0}$,

$$
\frac{\partial V_{\theta^{\star}}}{\partial t}(t, x)+\frac{\partial V_{\theta^{\star}}}{\partial x}(t, x) f\left(t, x, \theta^{\star}\right) \leq-\alpha_{\delta}(|x|) .
$$

If, furthermore, for all $i \in \llbracket 1, n \rrbracket$, it holds that

$$
\begin{gathered}
\lim _{\delta \rightarrow 0} \underline{a}_{i}+\underline{b}_{i} \theta_{i}^{\star}(\delta)>0, \\
\bar{b}_{i} \neq 0 \quad \Rightarrow \quad \lim _{\delta \rightarrow 0} \theta_{i}^{\star}(\delta) \delta^{p}=0,
\end{gathered}
$$

then the system (15) is UGPAS on the parameter set $\Theta$.

Remark 3 (High gain) When $\theta$ represents control gains, these usually need to be enlarged in order to achieve a better precision, which makes (21) satisfied in most cases.

Remark 4 (Quadratic Lyapunov function) It should be stressed that (19) holds in particular for quadratic Lyapunov function $V_{\theta}(x)=x^{T} P(\theta) x$ where $P \in \mathbb{R}^{x \times n}$ can be decomposed as $P(\theta)=P_{1}+P_{2} \theta$, where $P_{1}, P_{2} \in$ $\mathbb{R}^{n \times n}$ are independent of $\theta$.

\subsection{Cascades of UGPAS systems}

Based on these preliminaries about UGPAS, we now present recent results for the study of cascade interconnections of UGPAS systems. More precisely, we consider cascaded systems of the form

$$
\begin{aligned}
& \dot{x}_{1}=f_{1}\left(t, x_{1}, \theta_{1}\right)+g(t, x, \theta) \\
& \dot{x}_{2}=f_{2}\left(t, x_{2}, \theta_{2}\right)
\end{aligned}
$$

where $x:=\left(x_{1}^{T}, x_{2}^{T}\right)^{T} \in \mathbb{R}^{n_{1}} \times \mathbb{R}^{n_{2}}, \theta:=\left(\theta_{1}^{T}, \theta_{2}^{T}\right)^{T} \in$ $\mathbb{R}^{m_{1}} \times \mathbb{R}^{m_{2}}, t \in \mathbb{R}_{\geq 0}, f_{1}, f_{2}$ and $g$ are locally Lipschitz in the state and satisfy the Carathéodory conditions for all considered $\theta$. Note that, compared to the results of Section 2 , the cascade structure is slightly more constrained as it imposes that the interconnection term $g(t, x, \theta)$ appears as an additive term in the nominal dynamics of the driven subsystem $\dot{x}_{1}=f_{1}\left(t, x_{1}, \theta_{1}\right)$. We further assume that this interconnection term is uniformly bounded both in time and in $\theta_{2}$ and vanishes with $x_{2}$ :

\section{Assumption 1 (Bound on the interconnection term)}

For any $\theta_{1} \in \Theta_{1}$, there exists a nondecreasing function $G_{\theta_{1}}$ and a class $\mathcal{K}$ function $\Psi_{\theta_{1}}$ such that, for all $\theta_{2} \in \Theta_{2}$, all $x \in \mathbb{R}^{n_{1}} \times \mathbb{R}^{n_{2}}$ and all $t \in \mathbb{R}_{\geq 0}$,

$$
|g(t, x, \theta)| \leq G_{\theta_{1}}(|x|) \Psi_{\theta_{1}}\left(\left|x_{2}\right|\right) .
$$

We also assume that each subsystem is UGPAS when considered independently, and that we explicitly know a Lyapunov function characterizing this property for the driven subsystem.

Assumption 2 (UGPAS driving) The driving subsystem (23b) is UGPAS on $\Theta_{2}$.

\section{Assumption 3 (UGPAS driven + growth restrictions)} Given any $\delta_{1}>0$, there exist a parameter $\theta_{1}^{\star}\left(\delta_{1}\right) \in \Theta_{1}$, a continuously differentiable Lyapunov function $V_{\delta_{1}}$, class $\mathcal{K}_{\infty}$ functions $\underline{\alpha}_{\delta_{1}}, \bar{\alpha}_{\delta_{1}}, \alpha_{\delta_{1}}$ and a continuous positive nondecreasing function $c_{\delta_{1}}$ such that, for all $x_{1} \in \mathbb{R}^{n_{1}} \backslash \mathcal{B}_{\delta_{1}}$ and all $t \in \mathbb{R}_{\geq 0}$,

$$
\begin{gathered}
\underline{\alpha}_{\delta_{1}}\left(\left|x_{1}\right|\right) \leq V_{\delta_{1}}\left(t, x_{1}\right) \leq \bar{\alpha}_{\delta_{1}}\left(\left|x_{1}\right|\right) \\
\frac{\partial V_{\delta_{1}}}{\partial t}+\frac{\partial V_{\delta_{1}}}{\partial x_{1}} f_{1}\left(t, x_{1}, \theta_{1}^{\star}\right) \leq-\alpha_{\delta_{1}}\left(\left|x_{1}\right|\right) \\
\left|\frac{\partial V_{\delta_{1}}}{\partial x_{1}}\left(t, x_{1}\right)\right| \leq c_{\delta_{1}}\left(\left|x_{1}\right|\right) \\
\lim _{\delta_{1} \rightarrow 0} \underline{\alpha}_{\delta_{1}}^{-1} \circ \bar{\alpha}_{\delta_{1}}\left(\delta_{1}\right)=0 .
\end{gathered}
$$

In addition, for the function $G_{\theta_{1}}$ of Assumption 1, it holds that, for each $\delta_{1}>0$ and as stends to $+\infty$,

$$
\begin{aligned}
c_{\delta_{1}}(s) G_{\theta_{1}^{\star}\left(\delta_{1}\right)}(s) & =\mathcal{O}\left(\alpha_{\delta_{1}} \circ \bar{\alpha}_{\delta_{1}}^{-1} \circ \underline{\alpha}_{\delta_{1}}(s)\right)(28 \mathrm{a}) \\
\alpha_{\delta_{1}}(s) & =\mathcal{O}\left(\bar{\alpha}_{\delta_{1}}(s)\right) .
\end{aligned}
$$

The specific assumptions (26) and (28) made on the gradient of the Lyapunov function for the driven subsystem (and thus on the growth of the interconnection term) actually guarantee that the solutions of the overall cascade remain bounded, which is a key issue in cascade reasoning $[35,32,37]$. Note that this strong feature is ensured based on algebraic considerations, which simplifies the analysis.

Based on these three assumptions, we state the following result, originally proved in [6].

Theorem 6 (UGPAS + UGPAS) Under Assumptions 1, 2 and 3 , the cascaded system (23) is UGPAS on the parameter set $\Theta_{1} \times \Theta_{2}$. 
This result was successfully applied to spacecraft formation control [17], underway ship replenishment [19], PID control of robot manipulators [8] and disturbance rejection by smooth control [6].

As Theorem 6 does not provide information on the Lyapunov function associated to the cascade (23), it was not yet possible to extend this result to multiple cascaded systems such as in Section 2.3.

\section{CONCLUDING REMARKS}

This paper has synthesized different recent results obtained by the authors in the field of robustness analysis of cascaded systems. Two different approaches were considered.

The first one aims at considering explicitly the effect of a disturbance $u$ on the overall behavior of cascaded systems, using the formalism of iISS. More precisely, sufficient conditions were provided under which a cascade composed of an iISS subsystem driven by a GAS one is itself GAS. Similar developments were made for cascades of iISS subsystems.

The second approach was concerned with the practical stability that can be guaranteed to cascaded subsystems when free parameters can be tuned in order to reject the effect of perturbations.

\section{ACKNOWLEDGEMENT}

The first author is grateful to the scientific committee of the SSD conference for giving him the opportunity to present these results as a keynote lecture.

The first author also warmly thanks David Angeli, as the results presented in Section 2 are the fruit of a collaboration with him.

This research was supported by a $\mathrm{PhD}$ fellowship of the French Ministry of Research.

\section{REFERENCES}

[1] D. Angeli and A. Astolfi. A tight small gain theorem for not necessarily ISS systems. In Proc. 44th. IEEE Conf. Decision Contr., Sevilla, Spain, 2005.

[2] D. Angeli, B. Ingalls, E.D. Sontag, and Y. Wang. Separation principles for input-output and integralinput-to-state stability. SIAM J. on Contr. and Opt., 43(1):256-276, 2004.

[3] D. Angeli, E.D. Sontag, and Y. Wang. A characterization of integral input to state stability. IEEE Trans. on Automat. Contr., 45:1082-1097, 2000.

[4] M. Arcak, D. Angeli, and E.D. Sontag. A unifying integral ISS framework for stability of nonlinear cascades. SIAM J. on Contr. and Opt., 40:888-1904, 2002.

[5] A. Chaillet and D. Angeli. Integral input to state stable systems in cascade. Systems and Control Letters, 57:519-527, July 2008.
[6] A. Chaillet and A. Loría. Uniform global practical asymptotic stability for non-autonomous cascaded systems. European Journal of Control, 12(6):595605, 2006.

[7] A. Chaillet and A. Loría. Uniform semiglobal practical asymptotic stability for non-autonomous cascaded systems and applications. Automatica, 44(2):337-347, 2008.

[8] A. Chaillet, A. Loría, and R. Kelly. Robustness of PID-controlled manipulators vis a vis actuator dynamics and external disturbances. Accepted at European Journal of Control, 6:563-576, 2007.

[9] J.M. Coron. Global asymptotic stabilization for controllable systems without drift. Math. of Cont. Sign. and Syst., 5:295-312, 1992.

[10] B. d'Andréa Novel and J. M. Coron. Exponential stabilization of an overhead crane with flexible cable via a back-stepping approach. Automatica, 36:587593, 2000.

[11] H. Ito. Stability criteria for interconnected iiss systems and iss systems using scaling of supply rates. In Proc. American Control Conference, pages 10551060, 2004.

[12] H. Ito. Explicit solutons to state-dependent scaling problems for interconnected iiss and iss nonlinear systems. In Proc. American Control Conference, pages 4131-4136, 2005.

[13] H. Ito and Z.P. Jiang. Nonlinear Small-Gain Condition Covering iISS Systems: Necessity and Sufficiency from a Lyapunov Perspective. In Proc. 45th. IEEE Conf. Decision Contr., pages 355-360, 2006.

[14] H. Khalil. Nonlinear systems. Prentice Hall, 3rd ed., New York, 2001.

[15] P. V. Kokotović and R. Marino. On vanishing stability regions in nonlinear systems with high-gain feedback. IEEE Trans. on Automat. Contr., 31(10):967970, 1986.

[16] R. Kristiansen, A. Loría, A. Chaillet, and P. J. Nicklasson. Adaptive output feedback control of spacecraft relative translation. In CDC 2006, San Diego, USA, December 2006.

[17] R. Kristiansen, A. Loría, A. Chaillet, and P. J. Nicklasson. Output feedback control of relative translation in a leader-follower spacecraft formation, chapter in Proceedings of the Workshop on Group Coordination and Cooperative Control, pages 131-151. Lecture Notes in Control and Information Sciences. Springer Verlag, Norway, 2006.

[18] M. Krstić, I. Kanellakopoulos, and P. Kokotović. Nonlinear and Adaptive control design. John Wiley \& Sons, Inc., New York, 1995. 
[19] E. Kyrkjebø, E. Panteley, A. Chaillet, and K. Pettersen. A virtual vehicle approach to underway replenishment, chapter in Proceedings of the Workshop on Group Coordination and Cooperative Control, pages 171-189. Lecture Notes in Control and Information Sciences. Springer Verlag, Troms $\varnothing$, Norway, 2006.

[20] D. S. Laila, D. Nešić, and A. Astolfi. Sampleddata control of nonlinear systems, chapter in Advanced topics in control systems theory - Lecture notes from FAP 2005. Control and Information sciences. Springer Verlag, London, 2006. A. Loría, F. Lamnabhi-Lagarrigue and E. Panteley, eds.

[21] F. Lamnabhi-Lagarrigue, A. Loría, and E. Panteley. Advanced topics in control systems theory - Lecture notes from FAP 2004. Control and Information sciences. Springer Verlag, 2005. ISBN 1-85233-923-3.

[22] A. A. J. Lefeber. Tracking control of nonlinear mechanical systems. PhD thesis, University of Twente, Enschede, The Netherlands, 2000.

[23] D. Liberzon. Nonlinear control with limited information. Submitted to Communications in Information Systems, June 2008. Roger Brockett Legacy special issue.

[24] D. Liberzon, E.D. Sontag, and Y. Wang. Universal construction of feedback laws achieving ISS and integral-ISS disturbance attenuation. Syst. \& Contr. Letters, 46:111-127, 2002.

[25] A. Loría, T. I. Fossen, and E. Panteley. A separation principle for dynamic positioning of ships: theoretical and experimental results. IEEE Trans. Contr. Syst. Technol., 8(2):332-344, 2000.

[26] F. Mazenc, R. Sepulchre, and M. Jankovic. Lyapunov functions for stable cascades and applications to global stabilization. IEEE Trans. on Automat. Contr., 44:1795-1800, 1999.

[27] A. N. Michel, R. K. Miller, and W. Tang. Lyapunov stability of interconnected systems: decomposition into strongly connected subsystems. IEEE Trans. Circuits Sys., 25:799-809, 1978.

[28] D. Nešić and A. Loría. On uniform asymptotic stability of time-varying parameterized discrete-time cascades. IEEE Trans. on Automat. Contr., 49(6):875887, June 2004.

[29] D. Nešić and A. R. Teel. Input-to-sate stability for nonlinear time-varying systems via averaging. Math. of Cont. Sign. and Syst., 14:257-280, 2001.

[30] R. Ortega, A. Loría and R. Kelly. A semiglobally stable output feedback $\mathrm{PI}^{2} \mathrm{D}$ regulator for robot manipulators. IEEE Trans. on Automat. Contr., 40(8):14321436, 1995.
[31] E. Panteley and A. Loría. On global uniform asymptotic stability of non linear time-varying non autonomous systems in cascade. Syst. \& Contr. Letters, 33(2):131-138, 1998.

[32] E. Panteley and A. Loría. Growth rate conditions for stability of cascaded time-varying systems. Automatica, 37(3):453-460, 2001.

[33] E. Panteley, A. Loría and A. Sokolov. Global uniform asymptotic stability of nonlinear nonautonomous systems: Application to a turbo-diesel engine. European J. of Contr., 5:107-115, 1999.

[34] E. Panteley and R. Ortega. Cascaded control of feedback interconnected systems: Application to robots with ac drives. In Proc. 7th Congreso Latinoamericano de Control Automatico, Buenos Aires, Argentina, Sept. 1996.

[35] P. Seibert and R. Suárez. Global stabilization of nonlinear cascaded systems. Syst. \& Contr. Letters, 14:347-352, 1990.

[36] R. Sepulchre. Are basins of attraction easy to enlarge by feedback? In Plenary lecture at Congress on theoretical and applied mechanics, Louvain-la-Neuve, Belgium, 2000.

[37] R. Sepulchre, M. Arcak, and A. R. Teel. Trading the stability of finite zeros for global stabilization of nonlinear cascade systems. IEEE Trans. on Automat. Contr., 47(3):521-525, 2002.

[38] E.D. Sontag. Remarks on stabilization and Input-toState stability. In Proc. 28th. IEEE Conf. Decision Contr, pages 1376-1378, 1989.

[39] E.D. Sontag. A “universal" construction of artstein's theorem on nonlinear stabilization. Syst. \& Contr. Letters, 13(2):117-123, 1989.

[40] E.D. Sontag. Comments on integral variants of ISS. Syst. \& Contr. Letters, 34:93-100, 1998.

[41] E.D. Sontag. Input to state stability: Basic concepts and results, chapter in Nonlinear and Optimal Control Theory, pages 163-220. Lecture Notes in Mathematics. Springer-Verlag, Berlin, 2006. P. Nistri and G. Stefani eds.

[42] E.D. Sontag and M. Krichman. An example of a GAS system which can be destabilized by an integrable perturbation. IEEE Trans. on Automat. Contr., 48(6):1046-1049, 2003.

[43] E.D. Sontag and A.R. Teel. Changing supply functions in input/state stable systems. IEEE Trans. on Automat. Contr., 40(8):1476-1478, 1995.

[44] D. Soro and R. Lozano. Semi-global practical stabilization of an underactuated surface vessel via nested saturation controller. In Proc. American Control Conference, pages 2006 - 2011, 2003. 
[45] H. J. Sussmann and P. V. Kokotović. The peaking phenomenon and the global stabilization of nonlinear systems. IEEE Trans. on Automat. Contr. 36(4):424-439, 1991.

[46] Y. Tan, D. Nešić, and I.M.Y. Mareels. On non-local stability properties of extremum seeking control. $\mathrm{Au}$ tomatica, 42:889-903, 2006.

[47] A. R. Teel and J. Hespanha. Examples of GES systems that can be driven to infinity by arbitrarily small additive decaying exponentials. IEEE Trans. on Automat. Contr., 49:1407-1410, 2004.

[48] A. R. Teel, L. Moreau, and D. Nešić. Input-to-state set stability of pulse width modulated controllers with disturbances. Syst. \& Contr. Letters, 51:23-32, 2004.

[49] A. R. Teel, J. Peuteman, and D. Aeyels. Semi-global practical stability and averaging. Syst. \& Contr. Letters, 37:329-334, 1999.

[50] M. Vidyasagar. Decomposition tecnhiques for largescale systems with nonadditive interactions: stability and stabilizability. IEEE Trans. on Automat. Contr., 25(4):773-779, 1980.

[51] T. Yoshizawa. Stability theory by Lyapunov's second method. The Mathematical Society of Japan, Tokyo, 1966. 